\title{
Influence of Transmission Line Pitch on Passive Interference
}

\author{
Lu Ying ${ }^{1}$, Zhang Jian gong ${ }^{2}$, Gan Zheyuan ${ }^{2}$, Zhao Zhibin ${ }^{1}$ \\ ${ }^{1}$ College of Electrical and Electronical, North China Electric University, Beijing 102206, China \\ ${ }^{2}$ Wuhan Branch of China Electric Power Research Institute, State Key Laboratory for Environmental Protection of Power Grid, Wuhan \\ 430074, China
}

\begin{abstract}
Passive interference of transmission lines to neighbouring radio stations may affect the effective reception and transmission of radio station signals. Therefore, it is the basis to accurately calculate the electromagnetic scattering of transmission lines including overhead wires and towers under the circumstance of electromagnetic waves from outside. Wherein for radio stations, the overhead conductor is the main factor that affects the radar detection because of the working mode of the radar and other factors. In this paper, in the general case of the application of moment method to carry out passive interference calculations, the overhead lines are assumed to be all the actual wire. The space electric field strength and RCS of the different pitch at different operating frequencies and spaces are obtained. The influence of pitch on passive interference of overhead conductors was analyzed. Thus providing a basis for modeling to save energy and reduce the effects of passive interference provides the modeling basis.
\end{abstract}

\section{Introduction}

In recent years, with the rapid development of science and technology, massive construction of infrastructure such as transmission lines and radio stations has led to a close distance between the above two[1].In this case, even if the transmission line is not live working, its huge metal structure will affect the reception or transmission of signals from nearby radio stations, which leads to the increasing electromagnetic radiation from transmission lines to radio stations[2-3]. Therefore the problem that must be considered in power line construction is how to determine the reasonable distance between transmission line and radio station or reduce the passive interference of them.

To solve this problem, the United States[4-6], Canada[7], Japan[8,9] and other countries have carried out related research on the influence of power lines on signals in different frequency bands such as MF, VHF/UHV[10-11], and $\mathrm{m}$ waves since 1960s. Which involves radio wide broadcast[7], radar navigation[6] and other fields[8].It is an important practice for the electric power industry to implement this strategic decision to develop ultra-high voltage power transmission, optimize the allocation of energy resources and improve the comprehensive social benefits[12].Based on the mechanism of electromagnetic radiation and energy saving, the passive interference of transmission line is generated by the scattering of transmission line.

\section{Passive interference calculation model for transmission lines}

\subsection{Modelling algorithm for overhead conductors}

Since the calculation of passive interference between transmission lines and adjacent radio stations is an open field electromagnetic field calculation problem, most research literatures use Moment Method (MOM) or the rapid multipole method (FMM) based on MOM. In this section, the actual wire is considered as a twisted wire structure, and the modelling method of the stranded wire is given.

\section{2 modelling method of actual strand}

The actual wire is a twisted wire structure as shown in Figure.1
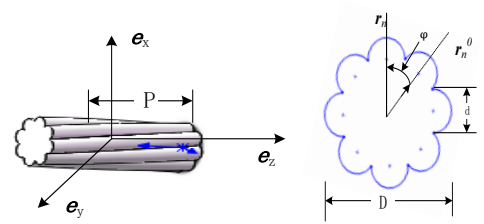

Fig.1 Geometry of a power line and its important parameters

The scattering electric field generated by such a strand also requires the use of a triangular face unit to split the surface. Therefore, the key to the modelling of the actual strand structure is the determination of the coordinates of the points on the surface of the strand. Any one of the sub-wires in the transmission line is composed of a number of steel strands (placed in the center) and a plurality of aluminum hinges (placed on the periphery). 
In order to establish a sub-conductor model, the $D$ is overall diameter of the sub-wire, $d$ is the overall diameter of sub-wires, $P$ is Peripheral aluminum strand diameter, $\varphi$ is an angle parameter slightly larger than $90^{\circ}$ between $\boldsymbol{r}_{\boldsymbol{n}}$ and $\boldsymbol{r}_{\boldsymbol{n}}^{0}, \boldsymbol{r}_{\boldsymbol{n}}$ and $\boldsymbol{r}_{\boldsymbol{n}}^{0}$ is the point and origin on the $n$ cycle of the sub-wire surface 。 According to the above parameters, a point on the surface of the wire can be expressed by the following formula [13]:

$$
\boldsymbol{r}=\boldsymbol{r}_{n}^{0}+\boldsymbol{r}_{n} \quad n=1,2, \ldots, N
$$

Where :

$$
\begin{aligned}
& \boldsymbol{r}_{n}{ }^{0}=\left(\frac{D-d}{2}\right) \cos \frac{2 \pi n}{N} \boldsymbol{e}_{x}+\left(\frac{D-d}{2}\right) \sin \frac{2 \pi n}{N} \boldsymbol{e}_{y} \\
& \boldsymbol{r}_{n}=\left(\frac{d}{2} \cos \frac{2 \pi n}{N} \cos \varphi-\frac{d \sqrt{(\pi D)^{2}+P^{2}}}{2 P} \times \sin \frac{2 \pi n}{N} \sin \varphi\right) \boldsymbol{e}_{x} \\
& +\left(\frac{d}{2} \sin \frac{2 \pi n}{N} \cos \varphi+\frac{d \sqrt{(\pi D)^{2}+P^{2}}}{2 P} \times \cos \frac{2 \pi n}{N} \sin \varphi\right) \boldsymbol{e}_{y}
\end{aligned}
$$

Table 1. Geometrical Specifications of Four Actual Power Lines

\begin{tabular}{c|c|c|c|c|c|c}
\hline \multirow{2}{*}{ NO } & \multicolumn{2}{|c|}{ Al/Copper } & \multicolumn{2}{|c|}{ Steel } & \multirow{2}{*}{$D(\mathrm{~cm})$} & \multirow{2}{*}{$P(\mathrm{~cm})$} \\
\cline { 2 - 5 } & \#of St. & $d(\mathrm{~cm})$ & \#of St. & $d(\mathrm{~cm})$ & & \\
\hline 1 & 7 & 0.400 & - & - & 1.20 & 14.60 \\
\hline 2 & 19 & 0.446 & - & - & 2.22 & 23.5 \\
\hline 3 & 54 & 0.337 & 7 & 0.337 & 3.01 & 35.56 \\
\hline 4 & 45 & 0.446 & 7 & 0.301 & 3.52 & 40.65 \\
\hline As
\end{tabular}
parameter representation of any point of the sub-wires can be expressed as

$$
R=\left[\boldsymbol{r}_{x} \cos \frac{2 \pi z}{P}-\boldsymbol{r}_{y} \sin \frac{2 \pi z}{P}, \boldsymbol{r}_{x} \sin \frac{2 \pi z}{P}+\boldsymbol{r}_{y} \cos \frac{2 \pi z}{P}, z\right]
$$

$\boldsymbol{r}_{x}$ and $\boldsymbol{r}_{y}$ are the components of $\boldsymbol{r}$, According to the above formula, the coordinates of any point on the outer surface of the power transmission line shown in Fig. 3 can be obtained for use in the division.

The twisted line model given in Fig. 3 is divided into triangular facets, and the electric field strength of each bin in space is the same as that of equation (5).

The calculation formula of the scattered electric field of the surface current of the line surface at any point in space is as shown in equation (5).

$$
\begin{aligned}
& \boldsymbol{E}_{S}(\boldsymbol{r})=j \omega \mu_{0} \bullet \int_{S} g\left(\boldsymbol{r}, \boldsymbol{r}^{\prime}\right) \boldsymbol{J}_{s}\left(\boldsymbol{r}^{\prime}\right) d S^{\prime} \\
& -\frac{1}{j \omega \varepsilon_{0}} \nabla \int_{S} g\left(\boldsymbol{r}, \boldsymbol{r}^{\prime}\right) \nabla^{\prime} \boldsymbol{J}_{s}\left(\boldsymbol{r}^{\prime}\right) d S^{\prime}
\end{aligned}
$$

$$
g\left(\boldsymbol{r}, \boldsymbol{r}^{\prime}\right) \text { is the Green's function, } g\left(\boldsymbol{r}, \boldsymbol{r}^{\prime}\right)=\frac{e^{j k\left|\boldsymbol{r}-\boldsymbol{r}^{\prime}\right|}}{4 \pi\left|\boldsymbol{r}-\boldsymbol{r}^{\prime}\right|} \text {; }
$$

$k$ is the Wave number, $k=\omega \sqrt{\mu_{0} \varepsilon_{0}}$.

In the calculation, in order to simulate the impact of overhead lines on radar operation, it is assumed that the electromagnetic wave direction $(\mathrm{k})$ emitted by the radar is perpendicular to the transmission line. Electric field strength (E) parallel to overhead transmission lines.

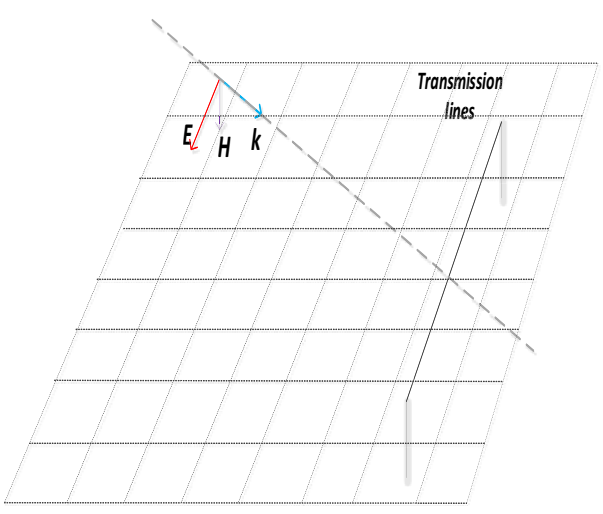

(a) Schematic diagram of the whole model

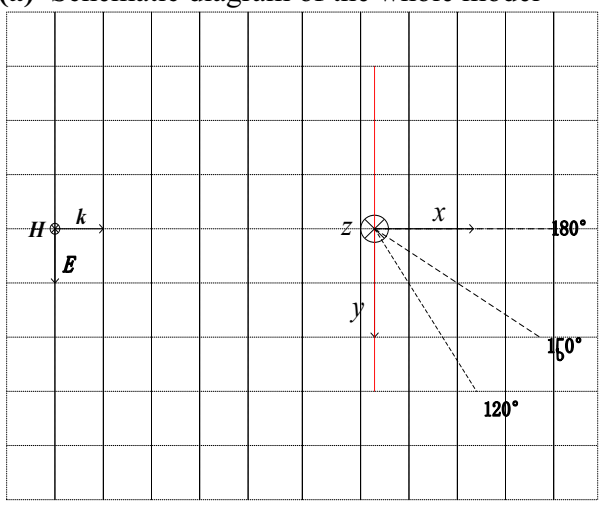

(b) The top view of the model

Fig.2 Passive interference computing model of overhead conductors

\section{Comparison of calculation results of spatial electric strength}

\subsection{Comparison of calculation results of spatial electric strength}

In order to compare different calculation models, the calculation results of the spatial electric field in different working frequencies of the radar and the distance from the transmission line, the maximum value of the electric field strength on the plane of different distances of the overhead line is taken to draw the curve shown in Fig. 3 in this section. In order to express the transmission line pitch more clearly, the pitch is replaced by the number of overhead wire windings. During the calculation, the horizontal distance between the observation point and the transmission line is $0.4 \mathrm{~km}, 0.6 \mathrm{~km}, 1.0 \mathrm{~km}$ and $2.0 \mathrm{~km}$, and the spatial electric field strength from $1 \mathrm{MHz}$ to $30 \mathrm{MHz}$ is calculated. 


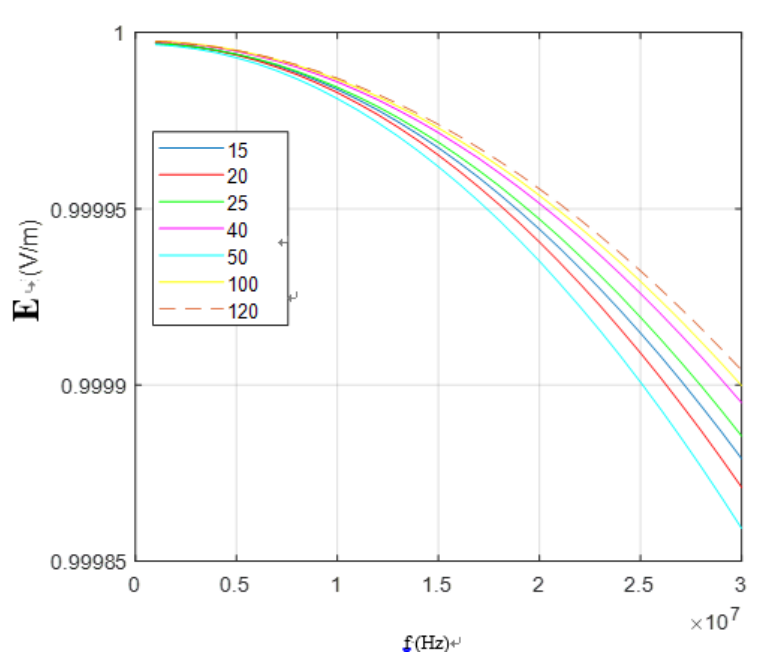

(a)The horizontal distance is $0.4 \mathrm{~km}$

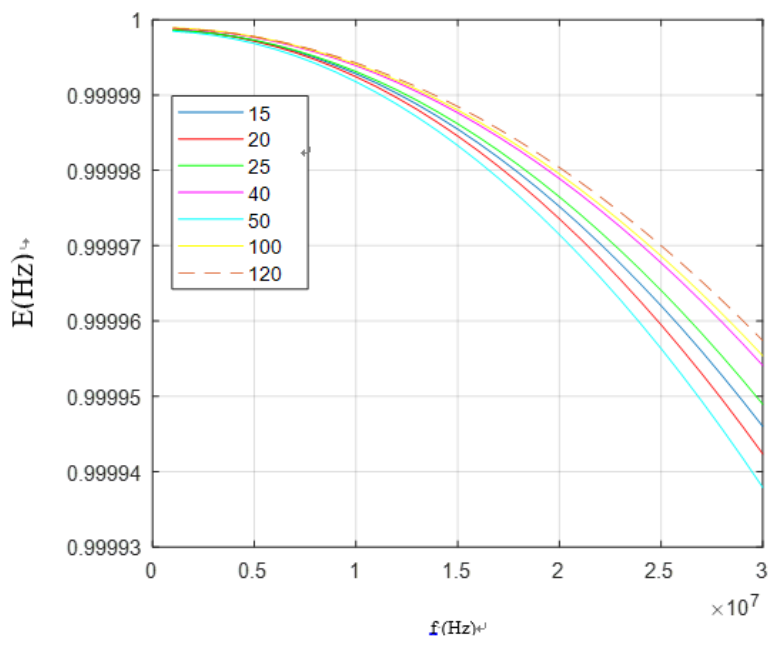

(b)The horizontal distance is $0.6 \mathrm{~km}$

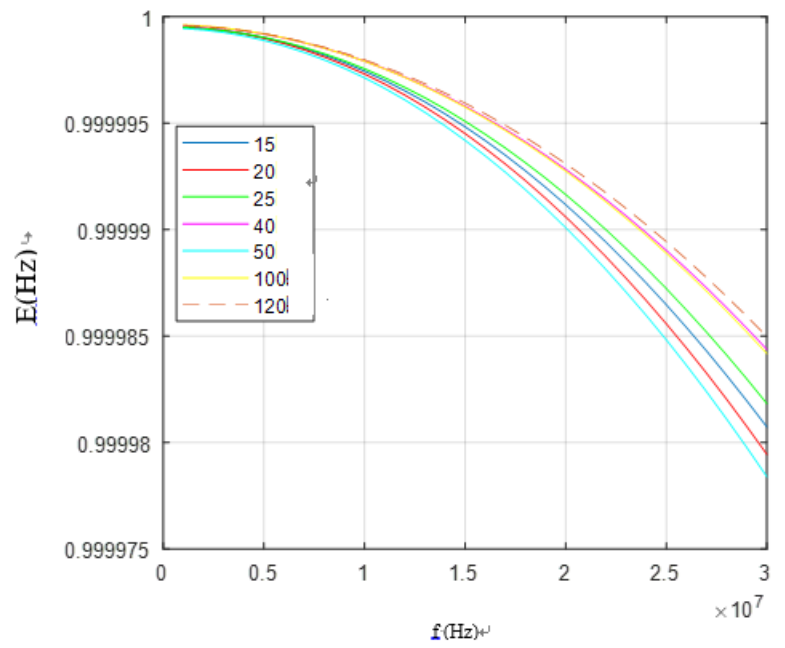

(c)The horizontal distance is $1.0 \mathrm{~km}$

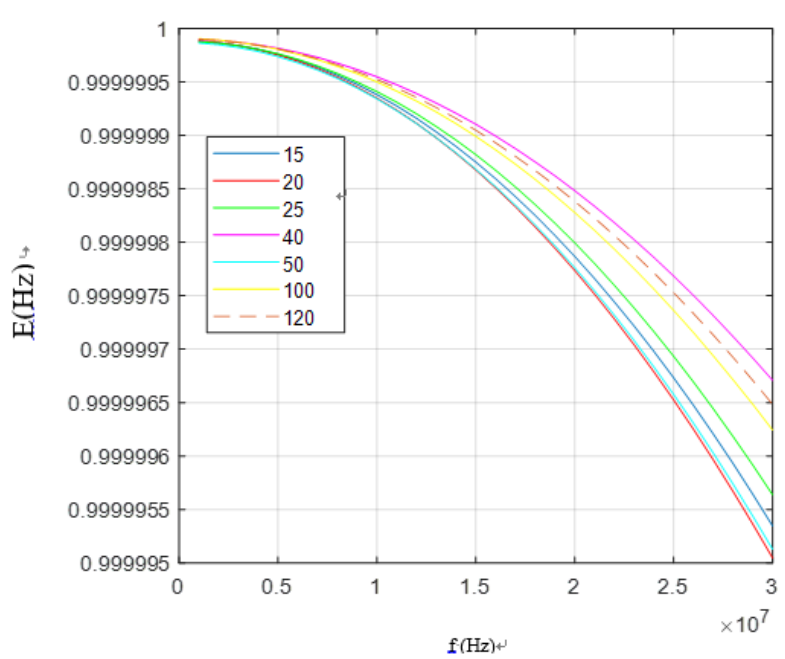

(d)The horizontal distance is $2.0 \mathrm{~km}$

Fig. 3 Calculation results of space electric field strength

The electric field strength generated by the actual transmission line is: $\operatorname{abs}\left(\boldsymbol{E}_{\mathrm{Fig}}-1\right)$ so, It is seen from the figure 3(a), (b), and (c), when the horizontal distance between the observation point and the transmission line is $0.4 \mathrm{~km}, 0.6 \mathrm{~km}$, and $1.0 \mathrm{~km}$, respectively, the electric field strength of the transmission line with 50 turns is the highest The electric field with 120 turns has the smallest. electric field strength; when the distance between the observation point and the transmission line is $2.0 \mathrm{~km}$, the electric field strength of the transmission line with 20 turns is the highest. the electric field with a lap of 40 and 120 produces the smallest electric field strength. In general, regardless of the trend of the pitch of the wire, with the frequency increasing, the electric field strength generated by the transmission line increases.

\subsection{Analysis of Relative Deviation of Overhead Conductors with Different Pitches}

In order to study the influence of the pitch of the transmission line on the passive interference, the electric field strengths of the transmission lines with different pitches are compared. Due to the limitations of the computer, the plane wave frequency range is set to the short wave range, and the parameters of the transmission line remain unchanged. The calculated electric field strength of the transmission line in the short-wavelength band is plotted as a function of the horizontal distance between the observation point and the transmission line (referred to as "horizontal distance") and the frequency. as shown in Figure 4, this section selects three sets of transmission lines with a difference of 20 pitches. Where the length of the overhead conductor is kept the same, Compare 1: the relative deviation of the electric field strength of 25 pitches and 5 pitches of the transmission line; compare2: the relative deviation of the electric field strength of 40 pitches and 20 pitches of the transmission line; compare 3: the relative deviation of the electric field strength of 120 pitches and 100 pitches of the transmission line . 


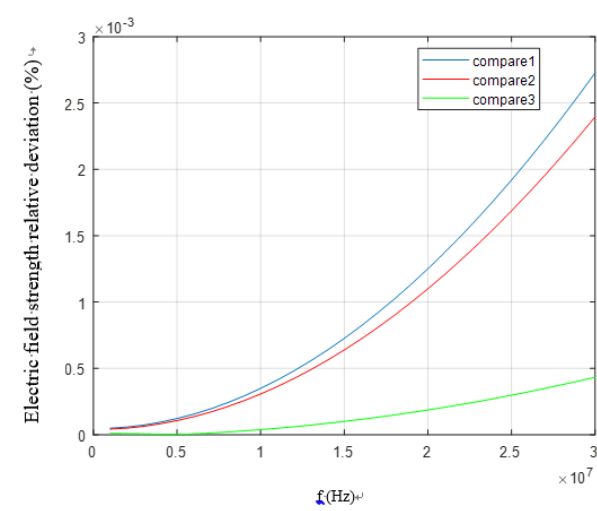

(a) The horizontal distance is $0.4 \mathrm{~km}$

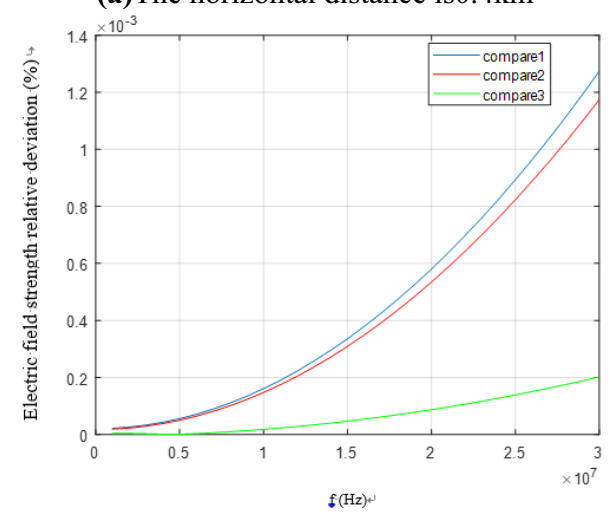

(b)The horizontal distance is $0.6 \mathrm{~km}$

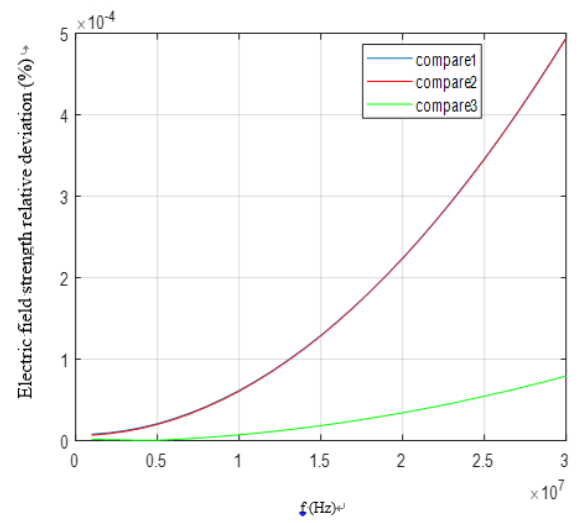

(c)The horizontal distance is $1.0 \mathrm{~km}$

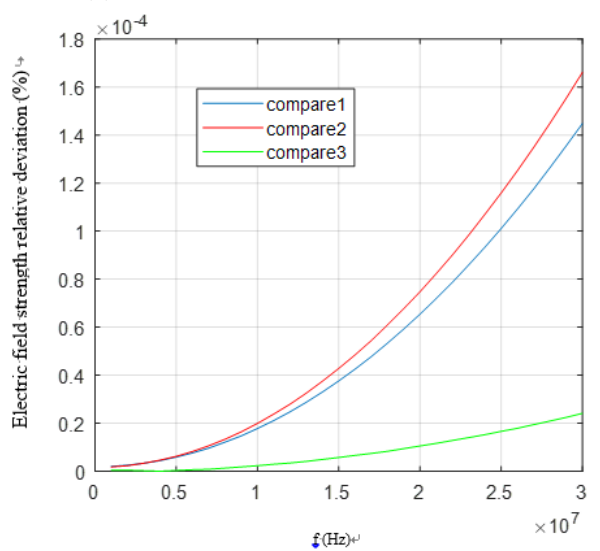

(d) The horizontal distance is $2.0 \mathrm{~km}$

Fig. 4 Schematic diagram of relative deviation of electric field intensity

It can be seen from Fig. 4 that in the range of $1 \mathrm{~km}$ distance, the relative deviation of the electric field strength of the 25 pitches and the five pitches of the transmission line is larger than the relative deviation of the electric field strength of the 40 pitches and 20 pitches of the transmission line; In the range of distance greater than $1 \mathrm{~km}$, the former is smaller than the latter; in addition, as the distance changes, the relative deviation of the electric field strength of the 120 pitch and 100 pitch of the transmission line is the smallest among the three groups. In general, as the horizontal distance increases, the relative deviation of the electric field strength generated by the transmission lines of different pitches is gradually decreasing. At the same time, as the frequency increases, the relative deviation of the electric field strength generated by the transmission lines of different pitches is gradually increasing.

\section{Conclusion}

In this paper, through simulation, the influence of transmission line pitch on passive interference in shortwavelength band is calculated, and the following conclusions are drawn, and the following conclusions are drawn.

1. Regardless of the trend of the pitch of the wire, as the distance between the observation point and the transmission line increases, the electric field strength generated by the transmission line decreases. At the same time, as the frequency increases, the electric field strength generated by the transmission line increases.

2. As the horizontal distance increases, the relative deviation of the electric field strength generated by the transmission lines of different pitches is gradually decreasing. At the same time, as the frequency increases, the relative deviation of the electric field strength generated by the transmission lines of different pitches gradually increases.

3. In the actual project, in order to reduce the influence of the passive interference of transmission line, within the distance of $1 \mathrm{~km}$ between the observation point and the transmission line, it is better to choose a $50 \mathrm{~m}$ overhead line with 40 pitches. Meanwhile, the goal of saving resources is achieved.

\section{References}

1. Yin hui, Zhang xiaoming, Wang yantao, et al. Research on 3D visualization of electromagnetic interference for Ultra-high Voltage transmission lines. High Voltage Engineering.40,12 (2014)

2. Tang bo, Yang jiawei Huang li, et al. Guard spacing of the air surveillance radar station to Ultrahigh Voltage transmission line.40.4(2018)

3. Tang bo, Sun rui, Zhao xiaoming, et al. Threedimensional Surface Computation model of the Reradiation interference From UHV angle-steel Tower[J]. Proceedings of the CSEE, 31, 4, 2011.

4. Wu xiong, Wan baoquan, Zhang xiaowu, et al. Study on the influence and protection of the radio station between the two circuits of the $1000 \mathrm{kV}$ high voltage exchange[R] .Wuhan, Hubei : Wuhan institute of high pressure, 2008. 
5. Hill P C.Measurement of reradiation from lattice masts at V.H,F.[J].Proc Inst Elec Eng,111,121964.

6. Belroses J S,Lavrench W,Dunn J G,et al.The effects of reradiation from high rise buildings and transmission lines upon the radiation pattern of $\mathrm{MF}$ broadcasing antenna arrays[C]//Proceedings of AGARD/EPP Meeting .Spatind,Norway:[s.n.],1979.

7. Kamal Sarabandi,Moonsooo Park.A radar crosssection model for power lines at millmeter-wave frequency[J].IEEE Transactions on Antenna and Propagation,51,9,2003.

8. Truemen C W, Kubina S J. Scatting from power lines with the skywire insulated from the towers[J].IEEE Transactions on Broadcasting,40,2,1994.

9. Li cheng, Wang qinjian, Liu youming. Research on passive interference from high-voltage transmission line affecting on medium wave navigation station[J].Modern electronics technique,39,7, 2016
10. Tang bo, Sun rui, Zhao xiaoming, et al. Passive interference solution for UHV transmission line in very high frequency band. Electric Power, 49, 10, 2016.

11. TANG Bo, WEN Yuanfang, ZHANG Xiaowu et al . Key problems of solving radiation interference protecting Distance Between Power transmission Line and Radio Station at MF and SF[J]. Proceedings of the CSEE, 31 ,19, 2011.

12. Sarabandi K, Park M. A Radar Cross-Section Model for Power Lines at Millimeter-Wave Frequencies [J]. IEEE Transactions on Antennas \& Propagation, 51, 9, 2003.

13. Kamal Sarabandi, A Radar Cross-Section Model for Power Lines at Millimeter-Wave Frequencies[J], IEEE Transactions on antennas and Propagation , 2003(9):2353-2360. 